\title{
Review Article \\ Periodontal Care as a Fundamental Step for an Active and Healthy Ageing
}

\section{Carlo Cafiero, Marco Matarasso, Gaetano Marenzi, Vincenzo Iorio Siciliano, Loredana Bellia, and Gilberto Sammartino}

University of Naples Federico II, Via Sergio Pansini 5, 80131 Napoli, Italy

Correspondence should be addressed to Carlo Cafiero; c.cafiero@unina.it

Received 14 October 2013; Accepted 25 November 2013

Academic Editors: A. Guentsch and F. Michon

Copyright (c) 2013 Carlo Cafiero et al. This is an open access article distributed under the Creative Commons Attribution License, which permits unrestricted use, distribution, and reproduction in any medium, provided the original work is properly cited.

\begin{abstract}
In the industrialized part of the world, an increasing number of people live the old age without too many restrictions due to illness or physiological impairment. This group is known as the young elderly. On the contrary, a consistent part of seniors develops a greater number of medical conditions and become more and more dependent, these are the old elderly. The first cause of tooth lost in industrialized word is periodontitis that generally strikes people older than 40 years and determines serious detriment of the stomatognatic organ. Smoking and stress are risk factors for periodontitis that are common and shared between young, adult, and older age. Diabetes mellitus, obesity, and osteoporosis are very frequent pathological situations in older age. They have been identified as cofactors in the progression of periodontitis. Many dental associations recognize the importance of continued research on oral fluids diagnostics and welcome the development of rapid point-of-care tests providing accurate measurements of clinically validated biomarkers. At present, well-studied molecules associated with host response factors and with derived tissue destruction mediators have been proposed as diagnostic biomarkers for periodontitis detected in the oral fluids.
\end{abstract}

\section{Introduction}

In the word, the elderly are an assorted population group whose number is growing at a considerably faster rate $(1.9 \%)$ than the total population (1.2\%). In the European Union (EU), the number of people aged $65+$ will almost double over the next 50 years, from 85 million in 2008 to 151 million in 2060 [1]. Being elderly has been held to be synonym to entering the third age: it lasts until the onset of the fourth age, that is characterized by deterioration, dependence, and ultimately death [2].

In the industrialized part of the world, an increasing number of people live this segment of life without too many restrictions due to illness or physiological impairment. This group is known as the young elderly. On the contrary, a consistent part of seniors develops a greater number of medical conditions and become more and more dependent. These are the old elderly [3].

Indeed, many conditions contribute to the frailty of the elderly, that is a condition making them more vulnerable to unfavourable health outcomes. Malnutrition, poor hydration, reduced physical activity, osteoporosis, and cognitive decline all contribute to poor health status. These conditions contribute to the occurrence, or to the evolution, of chronic diseases that are often present at the same time, and require complex poly-therapy regimens. In this context, poor prescription adherence is an additional component of frailty. The rising number of older people is linked to the growth of demands for health services and finally to unsustainable medical costs $[4,5]$.

While increased longevity is a great achievement, it is also a formidable challenge for both public and private budgets. For this reason, enlarging as much as possible the young elderly group appears fundamental, in order to have a healthy population and to lower nationals budgets covering the costs of Public Health. The European Commission has identified "active and healthy ageing" as a major social challenge, common to all the European countries. The pilot European Innovation Partnership on Active and Healthy Ageing (EIP$A H A)$ aims to enable EU citizens to lead healthy, active, and 
independent lives while ageing, by improving the sustainability and efficiency of social and health care systems. The all-encompassing target of this pilot partnership will be to increase the average healthy lifespan/lifetime by two years, by 2020 [6].

Prevention and early diagnosis of frailty and functional decline, both physical and cognitive, in older people is part of European Innovation Partnership on Active and Healthy Ageing 2020 initiative [7]. The old elderly group represents what we generally mean with the expression "to be old", that is, to be frail. One of the key determinants of frailty is malnutrition, that is very common in older people. Malnourished older people are at risk of experiencing fallings, prolonged hospitalization, postoperative complications, pressure ulcers, and death. Many causes of malnutrition have been proposed: metabolism not working properly, poverty, loss of taste sensations, mental depression, and difficulties in mastication of aliments. Periodontitis determines teeth loss and consequently difficulties in mastication of food. Furthermore, periodontitis represents a persistent, systemic inflammatory condition that contributes to the occurrence of acute events in the subjects with concurrent cardiovascular diseases.

For these reasons, we speculate that periodontitis might be considered as an additional risk factor for frailty in the elderly.

\section{Periodontitis. A Severe Infection of the Oral Cavity}

The first cause of tooth lost in industrialized word is periodontitis that generally strikes people older than 40 years. It is a genetically determined gram-negative anaerobic infection characterized by complex immune reactions to bacterial burden that determines formation of periodontal pockets subsequent connective attachment loss and bone resorption around the roots and might lead to tooth loss. Periodontitis is characterized by a cyclic progression in which a recurrent active phase (periodontal breakdown) is followed by a quiescence phase; appropriate therapy generally stops its progression. Periodontitis is classified into aggressive and chronic forms. Aggressive periodontitis (AP) is less common than the chronic form. Generally it affects younger patients causing rapid loss of attachment and bone destruction. The severity of periodontal tissue destruction is conflicting with the scarce amounts of microbial deposits. The reason of this destruction is the presence of elevated proportions of aggressive gram-negative bacteria, the phagocyte abnormalities and the hyperresponsive macrophage phenotype. Chronic periodontitis (CP) affects up to $50 \%$ of the global population, especially older patients. In most cases, the rate of progression of chronic periodontitis is slow, and the amount of periodontal tissue destruction is generally commensurate with subgingival calculus and plaque amounts. Both forms, Aggressive and Chronic, might determine severe destruction of periodontal tissues: for this reason periodontitis has to be definitely considered as a social disease, since it might determine serious detriment of stomatognatic organ, till reach the condition of edentulism [8].
2.1. Environmental Risk Factors for Periodontitis. Smoking and stress are risk factors for periodontitis that are common and shared between young, adult, and older age. Diabetes mellitus, obesity, and osteoporosis are very frequent pathological situations in older age. They have been identified as cofactors in the progression of periodontitis.

2.2. Smoking. Cigarette smoking is associated with a relative risk of developing periodontitis, ranging from 2.05 (95\% CI 1.47-2.87) for light smokers increasing to 4.75 (95\% CI 3.28-6.91) for heavy smokers. The negative effect of smoking is dose-dependent and cumulative and is associated with the recurrence of periodontitis after healing but during periodontal maintenance programme care [911]. Most studies have shown that in smokers less gingival bleeding is recorded than nonsmokers $[12,13]$. This situation could be subsequent to the vascular constriction determined by the nicotine effect. Moreover, the effect of smoking on periodontitis involve plaque microbiota composition, that determines higher prevalence of dangerous microbiota species (such as AA comitans, T. forsythia, and P. gingivalis), and scarce immunological reaction subsequently to impaired neutrophils and lymphocytes local reactions, as well as impaired fibroblast funcion.

2.3. Stress. Stress is currently considered as a risk indicator for periodontal disease. Stressful life events could affect periodontal disease progression through (1) unhealthy behaviours (poor oral hygiene, increased tobacco smoking) and (2) pathophysiological factors (higher glucocorticoid and catecholamine levels) which affect bacterial, immunological, inflammatory, and hormonal profiles, leading to an increased susceptibility to periodontal disease [14-16].

2.4. Diabetes Mellitus. Currently, the percentage of diabetics is very high worldwide, and these numbers are increasing dramatically. In the United States $8.3 \%$ of the population have diabetes. Between these, 10.9 million, or $26.9 \%$ of all people age 65 years or older have diabetes. These numbers mean that about one person upon four in elder group is affected by diabetes [17].

Patients with insulin-dependent and noninsulin-dependent diabetes mellitus have been found to be equally at risk of periodontitis [18]. It has been claimed that periodontitis is the sixth complication of diabetes, together with retinopathy, nephropathy, neuropathy, macrovascular diseases and altered wound healing [19]. Diabetes mellitus is the only systemic disease positively associated with attachment loss with an odds ratio of 2.32 (95\% confidence interval (CI) 1.17-4.60) [20]. Attachment loss and increased alveolar bone loss were found to be common in patients affected by uncontrolled diabetes (glycosylated hemoglobin $\geq 8 \mathrm{mg} / \mathrm{L}$ ).

The increased prevalence of periodontitis among diabetic patients has been attributed to the thickness augmentation of endothelial basement membrane, determining microangiopathy, as well as to neutrophils impaired function, hyper secretion of cytokines such as interleukin-1, interleukin-6, tumor necrosis factor-alpha, and prostaglandin E2. In particular, interleukin-6 appears to selectively suppress insulin 
action in hepatocytes. Some authors presumed a two-way relationship in which periodontal therapy can improve metabolic control in diabetic patients [21]. A recent Cochrane review on the treatment of periodontal disease for glycaemic control in people with diabetes declared that further controlled studies are necessary to clarify the topic [22].

2.5. Obesity. Obesity is defined as an unhealthy excess of body fat, which increases the risk of medical illness and premature mortality [23] The prevalence of obesity is increasing in all age groups, including older persons, defined as those $\geq 65$ years old [24]. With aging there is a greater relative increase in intra-abdominal fat than in subcutaneous fat. In addition, increases in intrahepatic fat in older persons are associated with insulin resistance [25]. It has been suggested that obesity is a strong risk factor for periodontal tissue destruction [26], since adipose tissue represents more than simple fat accumulation, it should be considered as an endocrine organ. It produces cytokines and hormones, collectively called adipokines or adipocytokines, which may play a key role in modulating periodontitis [27-31]. In addition, it was reported that maintaining a normal weight was associated with a poorer frequency of periodontitis $[32,33]$.

2.6. Osteoporosis. Due to its prevalence worldwide, osteoporosis is considered as a serious public health concern [34]. Currently it is estimated that over 200 million people worldwide suffer from this disease [35]. Approximately 30\% of all postmenopausal women have osteoporosis in the United States and in Europe. At least $40 \%$ of these women and $15-30 \%$ of men will experiment fragility fractures in lifetime [36]. Ageing of populations worldwide will be responsible for a major increase of the incidence of osteoporosis in postmenopausal women [37]. Osteoporosis is a metabolic bone disorder characterized by the loss of bone mineral density, principally recorded in postmenopausal women. It has been proposed that osteoporosis could affect the alveolar bone leading to rapid resorption in periodontal women, determining a positive relationship between osteoporosis and clinical attachment loss [38]. Other studies showed negative or equivocal results [39]. Thus, the association between osteoporosis and periodontitis in humans remains weak and still debatable [40].

2.7. Active and Healthy Aging. Early Diagnosis of Periodontitis as a Pathway to Get Young Elderly. Periodontitis has to be considered as a social disease since it affects millions of people in Europe as well as in the USA in which $31 \%$ of the population exhibited mild forms of periodontitis, $13 \%$ displayed periodontitis of moderate severity, and $4 \%$ suffered from advanced periodontitis [41]. Currently, accurate clinical periodontal diagnosis can be performed by periodontists, since they have sufficient diagnostic tools to get a clear picture of the actual single patient's periodontal conditions. Diagnostic imaging and periodontal charting provide a complete description of the patient's periodontal condition. By the use of a high-resolution professional digital camera, the operator takes a series of five pictures (frontal, right lateral, left lateral, palatal and lingual sides) that provides a clear picture of the patient's mouth. A full-mouth X-ray series is an important diagnostic support in periodontal patients (14/16 periapical $\mathrm{X}$-rays); it provides information on the height and configuration of the interproximal alveolar bone and the state of the patient's individual tooth from the crown to the tip of its root. Periodontal charting (full-mouth plaque score, full-mouth bleeding score, probing depth clinical attachment level, bleeding on probing, recessions, mobility, migration, halitosis) provides a complete picture of periodontal conditions of a single patient [42]. Probing pocket depth (PPD) allows an immediate evaluation of diseased sites (six sites for each tooth). It represents the distance from the gingival margin to the bottom of the gingival sulcus/pocket, at the mesiobuccal line angle, the midbuccal, the distobuccal line angle, the distolingual line angle, the midlingual, and the mesiolingual line.

The above described diagnosis is generally performed by periodontists, that are specialists. Unfortunately, most of the patients are visited by general dentists which do not provide such high level performances. Since early diagnosis of periodontitis is fundamental in order to stop at initial stage the progression of the disease, it appears necessary to organize a diagnostic platform allowing periodontal screening by general dentists. With the intention to detect periodontitis at an earlier stage, when it is easier to be treated successfully, several easy-to-use chair side tests have been proposed. The following points represent pioneeristic attempt to organize early diagnosis of periodontitis through the detection of bacterial species and biomarkers.

(i) Some microbiota are more important than others as etiological agents of periodontitis. Following the above criteria, the consensus report of the world workshop on periodontitis [43] identified three bacterial species for which sufficient data have accumulated as causative factors for periodontitis: Aggregatibacter actinomycetemcomitans, P. gingivalis, and Tannerella forsythia [44]. The consensus report stated that A. actinomycetemcomitans is most often found in aggressive ("early onset") periodontitis, whereas P. gingivalis and $\mathrm{T}$. forsythia are found more frequently in chronic ("adult-onset") periodontitis. Moderate evidence to support an aetiological P. nigrescens, Parvimonas micra (formerly Micromonas micros and Peptostreptococcus micros), the Streptococcus intermedius complex and T. denticola. Finally, an initial evidence included on the list of probable periodontal pathogens E. corrodens, enteric rods, Pseudomonas species, Selenomonas species, and Staphylococcus species. For these reasons, a DNA-based chair side test (semiquantitative polymerase chain reaction, PCR) of subgingival plaque could be a valid support for early diagnosis of periodontitis. Sampling of subgingival plaque by the insertion of sterile paper points into the deepest pockets in each quadrant are collected and send to a specialised laboratory that will perform the DNA examination and identification of bacterial species. 
(ii) In 1997, Kornman et al. described a composite genotype formed by two polymorphic loci-interleukin$1 \mathrm{~A}(-889)$ and interleukin-1B (+3954) - which are single-nucleotide polymorphisms that carry a C$\mathrm{T}$ transition. Interleukin-1 is a proinflammatory agent that is released by macrophages, lymphocytes, platelets, and endothelial cells. A sample of cells desquamating from the mucosa of the mouth allows examination to detect IL-1 polymorphism. A weak association between the single nucleotide Polymorphism in interleukin-1 genes and chronic periodontitis was found in a recent meta-analysis [45].

(iii) Many dental associations, such as the American Dental Association (ADA), recognise the importance of continued research on oral fluids diagnostics and welcome the development of rapid point-ofcare tests providing accurate measurements of clinically validated biomarkers. At present, well-studied molecules associated with host response factors and with derived tissue destruction mediators have been proposed as diagnostic biomarkers for periodontitis detected in the oral fluids. These components fall into three general categories: (1) host derived enzymes and their inhibitors, (2) inflammatory mediators, and host response modifiers and (3) tissue breakdown products.

We have searched the literature for more promising biomarker in regard to potential diagnostic value for periodontitis [46]. Consider the following.

(i) Alkaline phosphatase. Elevated alkaline phosphatase levels preceded periodontal destruction.

(ii) Beta-glucuronidase. It could be thought as an indicator of periodontal disease activity. Nakashima [47] reported that beta-glucuronidase was significantly higher in active versus inactive sites.

(iii) Cathepsin B. It is an enzyme active in proteolysis. Macrophages are the cellular source of cathepsin B in gingival crevicular fluid [48]. Cathepsin B levels (1) have been found to be increased in periodontitis but not in gingivitis [49-51].

(iv) Metalloproteinases-8. It appears 18-fold higher in progressing periodontitis versus stable periodontitis [52].

(v) Metalloprotheinases-9. It appears elevated in subjects affected by advanced periodontitis associated with red complex anaerobic periodontal pathogens (e.g., P. gingivalis and T. denticola) [53].

(vi) Dipeptidyl peptidases II and IV. Higher levels of both enzymes in sites with rapid and gradual attachment loss were reported with respect to sites without attachment loss [54].

(vii) Elastase. It has been recorded in oral fluid from periodontal patients at elevated levels and reduced after periodontal treatment [55-57]. (viii) $R A N K L / O P G / R A N K$ system. In the course of periodontitis, RANKL is secreted by osteoblasts, fibroblasts, bone marrow stromal cells, and activated $\mathrm{T}$ and B cells.

(ix) Pyridinoline cross-linked carboxyterminal telopeptide of type I collagen. High levels of 1-CTP were strongly correlated with clinical parameters and putative periodontal pathogens. Results showed that 1-CTP appeared as a good predictor of future alveolar bone and attachment loss and demonstrated significant reductions after periodontal therapy [58].

(x) Chondroitin-4-sulphate (C-4-S). It is the most common glycosaminoglycan in untreated chronic periodontitis [58]. A statistically significant correlation between the GCF content of C-4-S, a bone-specific glycosaminoglycan, and periodontal tissues destruction has been reported [59].

Even as gingival crevicular fluid (GCF), an exudate flushing from the gingival sulcus ( 0.5 to $2.5 \mathrm{~mL} / 24 \mathrm{~h}$ ) appears as the most appropriate diagnostic medium to use in analyses, it appears clear that the use of whole saliva is more practical even if reactants need to be highly sensitive since biomarkers are more diluted $[60,61]$.

\section{Conclusions}

More Europeans are surviving into old age. It appears inevitable that the longer people live, the more their capacity for self-care become reduced by physical or mental chronic diseases.

In order to gain active and healthy aging, it appears essential to create favorable condition allowing better access to health care in the younger years and consequently postponing the period of dependence to advanced age, so that the period with disability will be compressed [62].

It is not easy to reach this objective. As described above, smoking, stress, diabetes, obesity and osteoporosis represent risk factors for both general and periodontal health.

Oral fluid is the mirror of periodontal health. It is a medium for clinically relevant information since it contains biomarkers specific for periodontal diseases. Progresses in microfluidics technology are revolutionizing molecular biology procedures for enzymatic analysis, DNA analysis and proteomics. The evolution of microfluidics, digital microfluidics, appears promising for future application to diagnose periodontal diseases and to prognosticate periodontal treatment. Lab-on-a-chip (LOC) technology for periodontal inspection will involve less education than current diagnostic procedures and allow patients to be screened for periodontal disease in settings other than the periodontist practice, such as at general practitioners, general dentists or dental hygienists. All these benefits make the lab-on-a-chip technology ideal for predictive, preventive, personalized, and participatory periodontology recently defined by a paper as "the 5Ps age" [8]. On these basis, we speculate that periodontists, dentists and medical general practitioners should all together make an effort in order to remove risk factors for periodontal and general health. For a very long time dental operators 
have considered their patients as "big mouths moving on tiny legs". This wrong belief has led dentistry to abandon "mother house" general medicine and neglect fundamental aspects in diagnosis and therapy of several systemic disorders. Oral health has to be definitely considered as an important part of the human organism and not as an isolated segment. This appears like a "returning home" for dentistry. Finally, dental care is an important topic for healthy aging since a health stomatognatic apparatus is fundamental to get a good quality of life; first of all a good mastication means a good digestion and secondary food is an unequivocal mean helping in socialization of people, and socialization is a very important topic in old age for many reasons. Dental target of the immediate future is to allow elder people to arrive to advanced age with as much as possible functioning teeth in their mouth: this is the simple meaning of active and healthy aging in dentistry [63-65].

\section{Conflict of Interests}

The authors declare that there is no conflict of interests regarding the publication of this paper.

\section{References}

[1] http://ec.europa.eu/research/innovation-union/pdf/activehealthy-ageing/a3_action_plan.pdf.

[2] http://www.un.org/esa/population/publications/worldageing19502050.

[3] G. Mckenna and F. M. Burke, "Age-related oral changes," Dental update, vol. 37, no. 8, pp. 519-523, 2010.

[4] J. de Jong-Gierveld and H. van Solinge, Ageing and Its Consequences For the SocioMedical System. Population Studies, No 29.Strasbourg, Council of European Press, France, 1995.

[5] R. Holliday, "Ageing in the 21st century," The Lancet, vol. 354, no. 9196, p. SIV4, 1999.

[6] http://ec.europa.eu/research/innovation-union/index_en.cfm? section=active-healthy-ageing.

[7] http://ec.europa.eu/research/innovation-union/pdf/activehealthy-ageing/a3_action_plan.pdf.

[8] C. Cafiero and S. Matarasso, "Predictive, preventive, personalised and participatory periodontology: 'the 5Ps age' has already started," The EPMA Journal, vol. 4, 16 pages, 2013.

[9] M. S. Tonetti, "Cigarette smoking and periodontal diseases: etiology and management of disease," Annals of Periodontology, vol. 3, no. 1, pp. 88-101, 1998.

[10] G. Calsina, J.-M. Ramón, and J.-J. Echeverría, "Effects of smoking on periodontal tissues," Journal of Clinical Periodontology, vol. 29, no. 8, pp. 771-776, 2002.

[11] C. Rieder, A. Joss, and N. P. Lang, "Influence of compliance and smoking habits on the outcomes of supportive periodontal therapy (SPT) in a private practice," Oral Health Prev Dent, vol. 2, no. 2, pp. 89-94, 2004.

[12] G. Machuca, I. Rosales, J. R. Lacalle, C. Machuca, and P. Bullón, "Effect of cigarette smoking on periodontal status of healthy young adults," Journal of Periodontology, vol. 71, no. 1, pp. 7378, 2000 .

[13] R. M. Palmer, R. F. Wilson, A. S. Hasan, and D. A. Scott, "Mechanisms of action of environmental factors-tobacco smoking,"
Journal of Clinical Periodontology, vol. 32, no. 6, pp. 180-195, 2005.

[14] J. K. Kiecolt Glaser, D. Ricker, and J. George, "Urinary cortisol levels, cellular immunocompetency, and loneliness in psychiatric inpatients," Psychosomatic Medicine, vol. 46, no. 1, pp. 1523, 1984 .

[15] L. Boyapati and H.-L. Wang, "The role of stress in periodontal disease and wound healing," Periodontology 2000, vol. 44, no. 1, pp. 195-210, 2007.

[16] D. C. Peruzzo, B. B. Benatti, G. M. B. Ambrosano et al., "A systematic review of stress and psychological factors as possible risk factors for periodontal disease," Journal of Periodontology, vol. 78, no. 8, pp. 1491-1504, 2007.

[17] http://www.diabetes.org/diabetes-basics/diabetes-statistics/.

[18] T. Tervonen and R. C. Oliver, "Long-term control of diabetes mellitus and periodontitis," Journal of Clinical Periodontology, vol. 20, no. 6, pp. 431-435, 1993.

[19] H. Löe, "Periodontal disease: the sixth complication of diabetes mellitus," Diabetes Care, vol. 16, no. 1, pp. 329-334, 1993.

[20] S. G. Grossi, J. J. Zambon, A. W. Ho et al., "Assessment of risk for periodontal disease. I. Risk indicators for attachment loss," Journal of Periodontology, vol. 65, no. 3, pp. 260-267, 1994.

[21] S. G. Grossi and R. J. Genco, "Periodontal disease and diabetes mellitus: a two-way relationship," Annals of Periodontology, vol. 3, no. 1, pp. 51-61, 1998.

[22] T. C. Simpson, I. Needleman, S. H. Wild, D. R. Moles, and E. J. Mills, "Treatment of periodontal disease for glycaemic control in people with diabetes," Cochrane Database of Systematic Reviews, vol. 12, no. 5, 2010.

[23] D. T. Villareal, C. M. Apovian, R. F. Kushner, and S. Klein, "Obesity in older adults: technical review and position statement of the American Society for Nutrition and NAASO, The Obesity Society," American Journal of Clinical Nutrition, vol. 82, no. 5, pp. 923-934, 2005.

[24] J. A. Grinker, K. Tucker, P. S. Vokonas, and D. Rush, "Body habitus changes among adult males from the normative aging study: relations to aging, smoking history and alcohol intake," Obesity Research, vol. 3, no. 5, pp. 435-446, 1995.

[25] M. G. Cree, B. R. Newcomer, C. S. Katsanos et al., "Intramuscular and liver triglycerides are increased in the elderly," Journal of Clinical Endocrinology and Metabolism, vol. 89, no. 8, pp. 38643871, 2004

[26] C. S. Ritchie, “Obesity and periodontal disease," Periodontology 2000, vol. 44, no. 1, pp. 154-163, 2007.

[27] E. E. Kershaw and J. S. Flier, "Adipose tissue as an endocrine organ," Journal of Clinical Endocrinology and Metabolism, vol. 89, no. 6, pp. 2548-2556, 2004.

[28] T. Saito, Y. Shimazaki, and M. Sakamoto, "Obesity and periodontitis," The New England Journal of Medicine, vol. 339, no. 7, pp. 482-483, 1998.

[29] M. S. Al-Zahrani, N. F. Bissada, and E. A. Borawski, "Obesity and periodontal disease in young, middle-aged, and older adults," Journal of Periodontology, vol. 74, no. 5, pp. 610-615, 2003.

[30] G. Linden, C. Patterson, A. Evans, and F. Kee, "Obesity and periodontitis in 60-70-year-old men," Journal of Clinical Periodontology, vol. 34, no. 6, pp. 461-466, 2007.

[31] R. J. Genco, S. G. Grossi, A. Ho, F. Nishimura, and Y. Murayama, "A proposed model linking inflammation to obesity, diabetes, and periodontal infections," Journal of Periodontology, vol. 76, no. 11, pp. 2075-2084, 2005. 
[32] M. S. Al-Zahrani, E. A. Borawski, and N. F. Bissada, "Increased physical activity reduces prevalence of periodontitis," Journal of Dentistry, vol. 33, no. 9, pp. 703-710, 2005.

[33] M. S. Al-Zahrani, E. A. Borawski, and N. F. Bissada, "Periodontitis and three health-enhancing behaviors: maintaining normal weight, engaging in recommended level of exercise, and consuming a high-quality diet," Journal of Periodontology, vol. 76, no. 8, pp. 1362-1366, 2005.

[34] http://www.iofbonehealth.org/epidemiology.

[35] C. Cooper, G. Campion, and L. J. Melton III, "Hip fractures in the elderly: a world-wide projection," Osteoporosis International, vol. 2, no. 6, pp. 285-289, 1992.

[36] A. Randell, P. N. Sambrook, T. V. Nguyen et al., "Direct clinical and welfare costs of osteoporotic fractures in elderly men and women," Osteoporosis International, vol. 5, no. 6, pp. 427-432, 1995.

[37] J.-Y. Reginster and N. Burlet, "Osteoporosis: a still increasing prevalence," Bone, vol. 38, no. 2, pp. S4-S9, 2006.

[38] J. Wactawski-Wende, "Periodontal diseases and osteoporosis: association and mechanisms," Annals of Periodontology, vol. 6, no. 1, pp. 197-208, 2001.

[39] N. Von Wowern, B. Klausen, and K. Olgaard, "Steroid-induced mandibular bone loss in relation to marginal periodontal changes," Journal of Clinical Periodontology, vol. 19, no. 3, pp. 182-186, 1992.

[40] A. Yoshihara, Y. Seida, N. Hanada, and H. Miyazaki, "A longitudinal study of the relationship between periodontal disease and bone mineral density in community-dwelling older adults," Journal of Clinical Periodontology, vol. 31, no. 8, pp. 680-684, 2004.

[41] J. M. Albandar, J. A. Brunelle, and A. Kingman, "Destructive periodontal disease in adults 30 years of age and older in the United States, 1988-1994," Journal of Periodontology, vol. 70, no. 1, pp. 13-29, 1999.

[42] J. Lindhe, N. P. Lang, and T. Karring :, "The anatomy of periodontal tissue," in Clinical Periodontology and Implant Dentistry, vol. 1, pp. 3-48, Wiley-Blackwell, 5th edition, 2008.

[43] N. Nørskov-Lauritsen and M. Kilian, "Reclassification of Actinobacillus actinomycetemcomitans, Haemophilus aphrophilus, Haemophilus paraphrophilus and Haemophilus segnis as Aggregatibacter actinomycetemcomitans gen. nov., comb. nov., Aggregatibacter aphrophilus comb. nov. and Aggregatibacter segnis comb. nov., and emended description of Aggregatibacter aphrophilus to include $\mathrm{V}$ factor-dependent and $\mathrm{V}$ factorindependent isolates," International Journal of Systematic and Evolutionary Microbiology, vol. 56, no. 9, pp. 2135-2146, 2006.

[44] M. Sakamoto, M. Suzuki, M. Umeda, I. Ishikawa, and Y. Benno, "Reclassification of Bacteroides forsythus as Tanerella forsythensis corrig., gen. nov., comb. nov," International Journal of Systematic and Evolutionary Microbiology, vol. 52, no. 3, pp. 841-849, 2002.

[45] B. G. Loos, F. G. J. Leppers-Van De Straat, J. G. J. Van De Winkel, and U. Van Der Velden, "Fc $\gamma$ receptor polymorphisms in relation to periodontitis," Journal of Clinical Periodontology, vol. 30, no. 7, pp. 595-602, 2003.

[46] B. G. Loos and S. Tjoa, "Host-derived diagnostic markers for periodontitis: do they exist in gingival crevice fluid?" Periodontology 2000, vol. 39, pp. 53-72, 2005.

[47] K. Nakashima, "A longitudinal study of various crevicular fluid components as markers of periodontal disease activity," Journal of Clinical Periodontology, vol. 23, no. 9, pp. 832-838, 1996.
[48] C. N. Kennett, S. W. Cox, and B. M. Eley, "Investigations into the cellular contribution to host tissue proteases and inhibitors in gingival crevicular fluid," Journal of Clinical Periodontology, vol. 24, no. 6, pp. 424-431, 1997.

[49] B. M. Eley and S. W. Cox, "The relationship between gingival crevicular fluid cathepsin B activity and periodontal attachment loss in chronic periodontitis patients: a 2-year longitudinal study," Journal of Periodontal Research, vol. 31, no. 6, pp. 381392, 1996.

[50] H. Y. Chen, S. W. Cox, and B. M. Eley, "Cathepsin B, $\alpha 2-$ macroglobulin and cystatin levels in gingival crevicular fluid from chronic periodontitis patients," Journal of Clinical Periodontology, vol. 25, no. 1, pp. 34-41, 1998.

[51] S. W. Cox and B. M. Eley, "Cathepsin B/L-, elastase-, tryptase-, trypsin- and dipeptidyl peptidase IV-like activities in gingival crevicular fluid. A comparison of levels before and after basic periodontal treatment of chronic periodontitis patients," Journal of Clinical Periodontology, vol. 19, no. 5, pp. 333-339, 1992.

[52] C. A. Ramseier, J. S. Kinney, A. E. Herr et al., "Identification of pathogen and host-response markers correlated with periodontal disease," Journal of Periodontology, vol. 80, no. 3, pp. 436446, 2009.

[53] W. V. Giannobile, "Salivary diagnostics for periodontal diseases," The Journal of the American Dental Association, vol. 143, pp. 6S-11S, 2012.

[54] B. M. Eley and S. W. Cox, "Correlation between gingival crevicular fluid dipeptidyl peptidase II and IV activity and periodontal attachment loss. A 2-year longitudinal study in chronic periodontitis patients," Oral Diseases, vol. 1, no. 4, pp. 201-213, 1995.

[55] L. Jin, B. Söder, and E. F. Corbet, "Interleukin-8 and granulocyte elastase in gingival crevicular fluid in relation to periodontopathogens in untreated adult periodontitis," Journal of Periodontology, vol. 71, no. 6, pp. 929-939, 2000.

[56] Q. T. Smith, L. Harriman, G. S. Au et al., "Neutrophil elastase in crevicular fluid: comparison of a middle-aged general population with healthy and periodontitis groups," Journal of clinical periodontology, vol. 22, no. 12, pp. 935-941, 1995.

[57] K. G. Palcanis, I. K. Larjava, B. R. Wells et al., "Elastase as an indicator of periodontal disease progression," Journal of Periodontology, vol. 63, no. 4, pp. 237-242, 1992.

[58] W. V. Giannobile, K. F. Al-Shammari, and D. P. Sarment, "Matrix molecules and growth factors as indicators of periodontal disease activity," Periodontology 2000, vol. 31, pp. 125-134, 2003.

[59] A. J. Smith, M. Addy, and G. Embery, "Gingival crevicular fluid glycosaminoglycan levels in patients with chronic adult periodontitis," Journal of Clinical Periodontology, vol. 22, no. 5, pp. 355-361, 1995.

[60] I. L. C. Chapple, "Periodontal disease diagnosis: current status and future developments," Journal of Dentistry, vol. 25, no. 1, pp. 3-15, 1997.

[61] I. L. Chapple, J. B. Matthews, G. H. Thorpe, H. D. Glenwright, J. M. Smith, and M. S. Saxby, "A new ultrasensitive chemiluminescent assay for the site-specific quantification of alkaline phosphatase in gingival crevicular fluid," Journal of Periodontal Research, vol. 28, no. 4, pp. 266-273, 1993.

[62] M. Södergren, "Lifestyle predictors of healthy ageing in men," Maturitas, vol. 75, no. 2, pp. 113-117, 2013.

[63] O. Golubnitschaja, "Time for new guidelines in advanced diabetes care: paradigm change from delayed interventional approach to predictive, preventive \& personalized medicine," EPMA Journal, vol. 1, no. 1, pp. 3-12, 2010. 
[64] O. Golubnitschaja and V. Costigliola, "European strategies in predictive, preventive and personalised medicine: highlights of the EPMA World Congress 2011," EPMA Journal, vol. 2, no. 4, pp. 315-332, 2011.

[65] O. Golubnitschaja, I. D. Watson, E. Topic, S. Sandberg, M. Ferrari, and V. Costigliola, "Position paper of the EPMA and EFLM: a global vision of the consolidated promotion of an integrative medical approach to advance health care," EPMA Journal, vol. 4, no. 1, p. 12, 2013. 


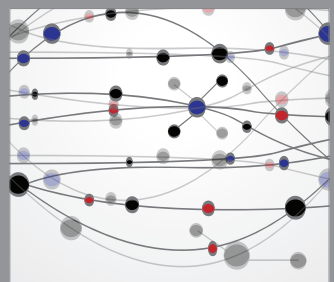

The Scientific World Journal
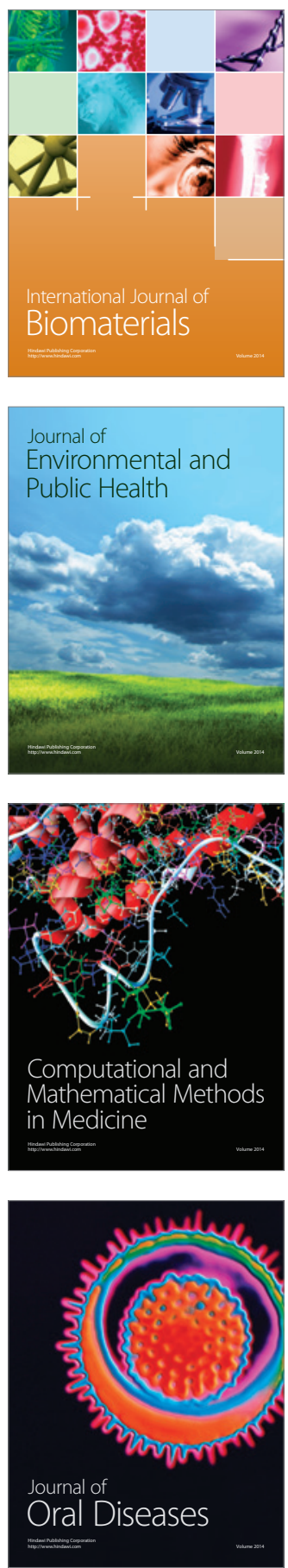
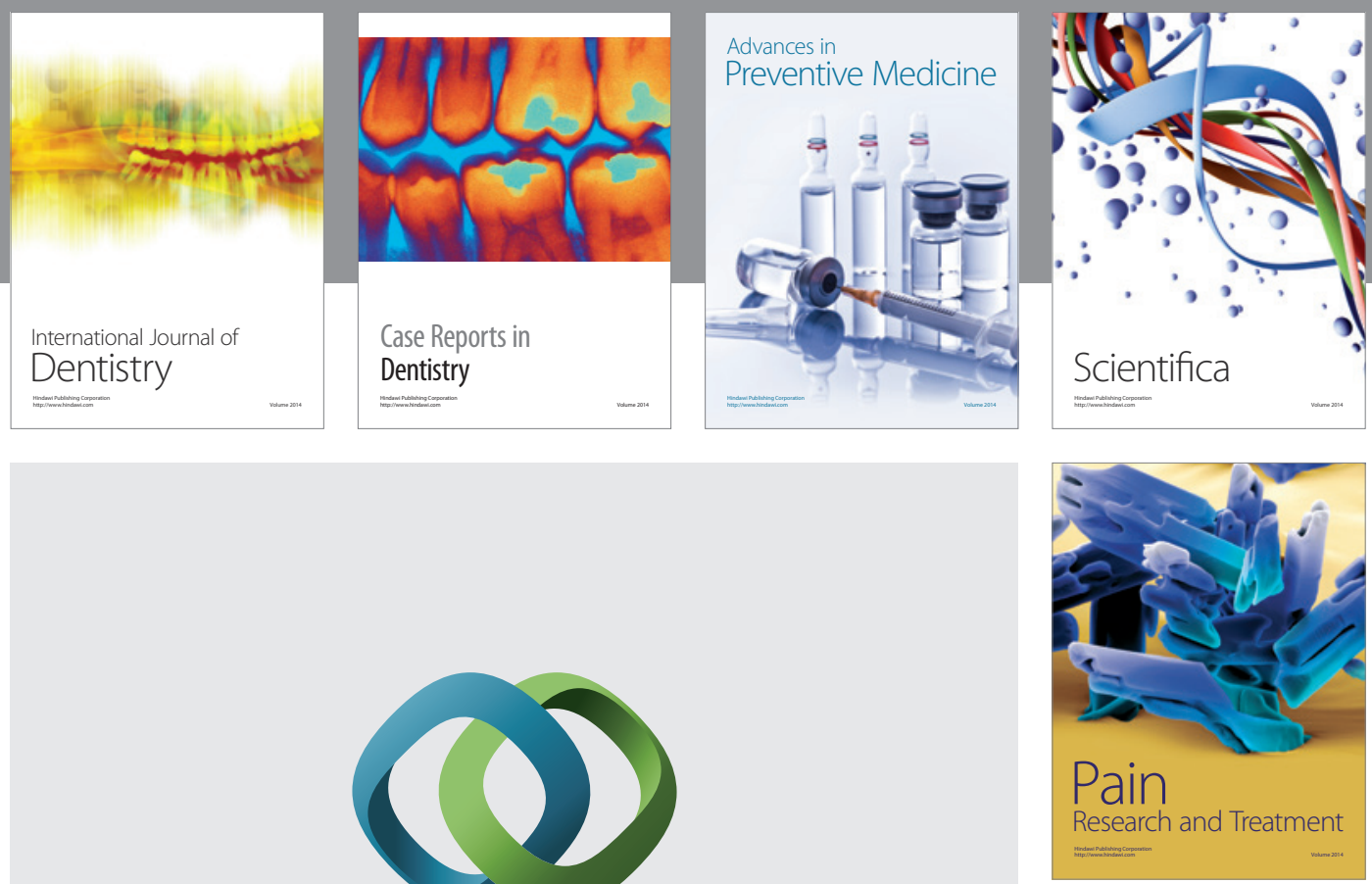

\section{Hindawi}

Submit your manuscripts at

http://www.hindawi.com
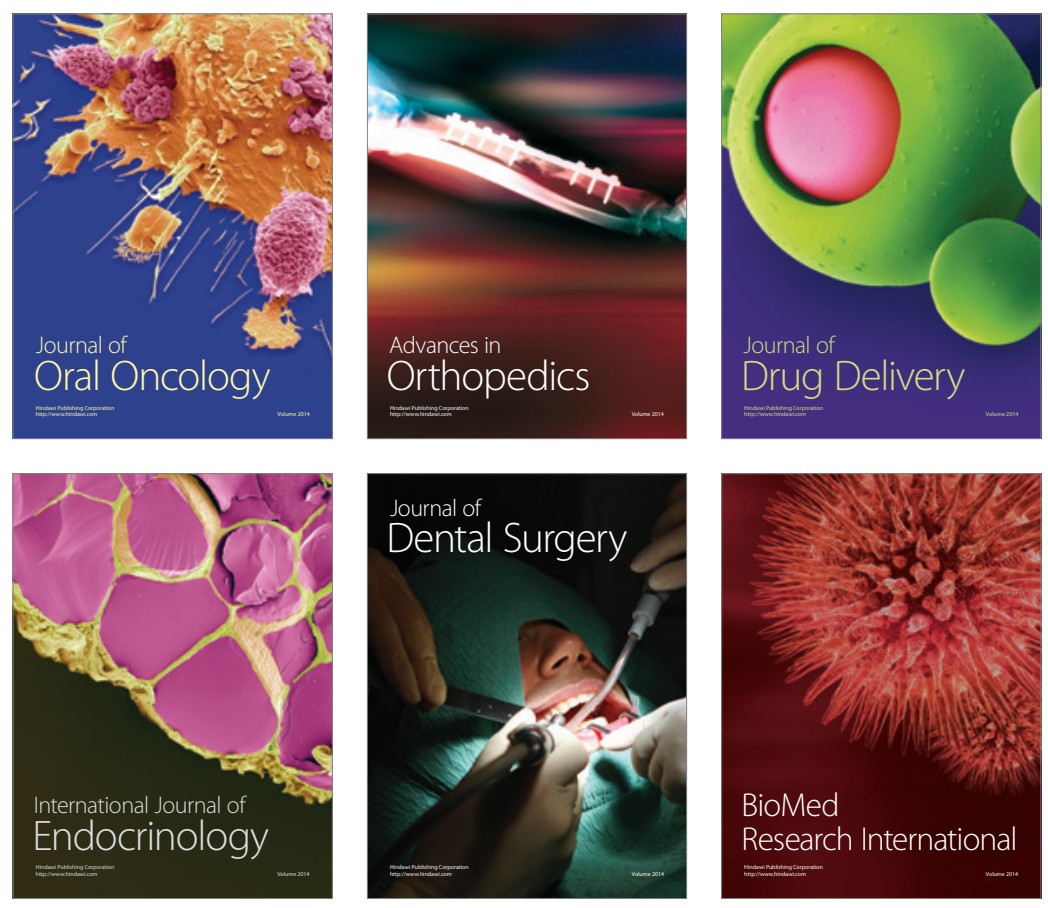

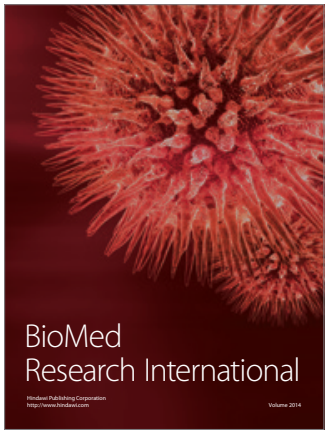

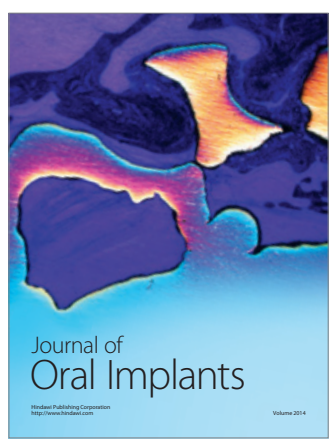
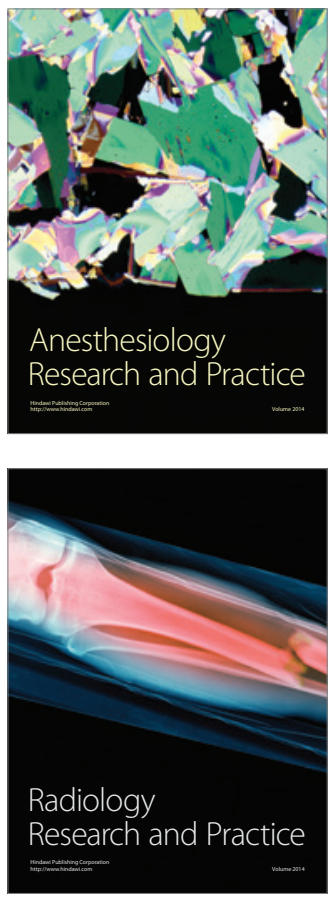